\title{
Quorum sensing inhibition as a promising method to control biofilm growth in metalworking fluids
}

\section{Authors: Safiye S. Ozcan, Markus Dieser, Albert E. Parker, Narayanaganesh Balasubramanian, and Christine M. Foreman}

The final publication is available at Springer via http://dx.doi.org/10.1007/s10295-019-02181-7.

Ozcan, Safiye S., Markus Dieser, Albert E. Parker, Narayanaganesh Balasubramanian, and Christine M. Foreman. "Quorum sensing inhibition as a promising method to control biofilm growth in metalworking fluids." Journal of Industrial Microbiology \& Biotechnology (April 2019): 1-9. DOI:10.1007/s10295-019-02181-7. 


\section{QUORUM SENSING INHIBITION AS A PROMISING METHOD TO CONTROL BIOFILM GROWTH IN METALWORKING FLUIDS}

Safiye Selen Özcan ${ }^{1,3}$, Markus Dieser ${ }^{1,3}$, Albert E. Parker ${ }^{3,4}$, Narayanaganesh Balasubramanian ${ }^{2}$, Christine M. Foreman ${ }^{1,3, *}$

${ }^{1}$ Department of Chemical \& Biological Engineering, Montana State University, Bozeman, MT 59715

${ }^{2}$ Department of Chemistry and Biochemistry, Montana State University, Bozeman, MT 59717

${ }^{3}$ Center for Biofilm Engineering, Montana State University, Bozeman, MT 59717

${ }^{4}$ Department of Mathematical Sciences, Montana State University, Bozeman, MT 59717

\section{* Corresponding Author: \\ Montana State University \\ Center for Biofilm Engineering \\ 366 Barnard Hall \\ Bozeman, MT 59717, USA \\ Email: cforeman@montana.edu}

Phone: 406-994-7361

Draft for submission to Journal of Industrial Microbiology and Biotechnology

Keywords: Metalworking fluids, biofilm, quorum sensing inhibition, patulin, furanone C-30 


\section{Acknowledgments}

The authors would like to thank members of the Center for Biofilm Engineering for sharing their expertise in growing and imaging biofilms; in particular Laura Boegli and Betsey Pitts. Funding was provided by National Science Foundation (NSF 1635347). Funding for the Proteomics, Metabolomics and Mass Spectrometry Facility was made possible in part by the MJ Murdock Charitable Trust and the National Institute of General Medical Sciences of the National Institutes of Health (P20GM103474). Comments of two anonymous reviewers helped strengthen the final manuscript. Any opinions, findings, or conclusions expressed in this material are those of the authors and do not necessarily reflect the views of the funding agencies. 


\begin{abstract}
Microbial contamination in metalworking systems is a critical problem. This study determined the microbial communities in metalworking fluids (MWFs) from two machining shops and investigated the effect of quorum sensing inhibition (QSI) on biofilm growth. In both operations, biofilm-associated and planktonic microbial communities were dominated by Pseudomonadales (60.2-99.7\%). Rapid recolonization was observed even after dumping spent MWFs and meticulous cleaning. Using Pseudomonas aeruginosa PAO1 as a model biofilm organism, patulin $(40 \mu \mathrm{M})$ and furanone C-30 $(75 \mu \mathrm{M})$ were identified as effective QSI agents. Both agents had a substantially higher efficacy compared to $\alpha$-amylase (extracellular polymeric substance degrading enzyme) and reduced biofilm formation by $63 \%$ and $76 \%$ respectively in MWF when compared to untreated controls. Reduced production of putatively identified homoserine lactones and quinoline in MWF treated with QS inhibitors support the effect of QSI on biofilm formation. The results highlight the effectiveness of QSI as a potential strategy to eradicate biofilms in MWFs.
\end{abstract}




\section{Introduction}

Metalworking fluids (MWF) are used in machining, grinding, and milling operations for cooling, reducing friction, removing metal particles, and protecting the workpiece and tools. Microbial contamination of MWFs is a fundamental problem, affecting the quality of the product, the lifetime of MWFs [13, 48], and potentially increasing occupational safety risks [6]. Measures to combat microbial growth in MWFs typically involve the use of biocides, although alternative, more environmentally friendly approaches have also been investigated $[1,2]$. These measures are, nonetheless, insufficient at eliminating the microbial load in MWF circulation systems [14, 23, 27, 50]. In fact, certain bacterial strains present in MWFs (e.g. Mycobacterium and Pseudomonas) are particularly resistant to industrial biocides [11, 48, 49]. Rapid recolonization of freshly recharged MWF systems directly after emptying and extensive cleaning is a strong indication of the ineffective removal of microbial colonizers $[18,50]$. The microbial contaminants are believed to persist in the inaccessible parts of these machines in the form of biofilms [48]. While increasing biocide dosage could potentially improve biofilm disruption in MWFs [24], such attempts would be counterproductive to current trends in the MWF industry, by raising costs, increasing occupational safety and environmental risks, and impeding the biodegradation of spent MWFs.

Intercellular communication, a phenomenon termed quorum sensing (QS), has been identified across diverse bacterial taxa and enables complex interactions among bacteria [28]. Along with other physiological processes, the formation of biofilm, a well-organized and structured microbial community enclosed in a self-produced polymeric matrix and adherent to a surface, is regulated by QS [29]. Fundamentally, QS is mediated by the production, release, sensing of, and response to small, diffusible signal molecules by bacterial cells (i.e. 
autoinducers), and is related to cell density [28, 32]. Once the threshold concentration for signaling molecules is reached in the extracellular environment, these molecules diffuse through cell membranes and activate transcription regulators [28]. This dependency on signal molecules to coordinate a translational behavior on a population level provides ideal targets to disrupt bacterial communication and, hence, biofilm formation. While QS systems and their inhibition [e.g. 12, 16, 41], by numerous synthetic $[14,31,39,51]$ and naturally occurring QS disruptive compounds $[9,14,17,21,39,40,46]$ have been studied extensively, limited effort has been directed to the application of QSI [reviewed in 16, 42]. However, some large-scale trials on water treatment systems have demonstrated the applicability of QSI to successfully mitigate biofilm formation and biofouling [19, 22, 30, 44].

Considering that bacteria resistant to biocides are emerging among microbial communities inhabiting MWF circulation systems [11, 48, 49], we assessed whether QSI, a nonbiocide treatment, can reduce biofilm formation in these systems. The effect of commercially available QSI compounds on biofilm reduction in a water soluble MWF were investigated. With Pseudomonas spp. frequently being the focus of research on QSI [e.g. 14, 21, 39, 40, 45, 51] as well as representing the bacterial genus that dominates microbial contamination in MFW [10, 13, 27], Pseudomonas aeruginosa PAO1 was used as a model organism in the present study.

\section{Materials and Methods}

Sample collection

Planktonic and biofilm samples were collected from computer numerical control (CNC) machines from two local machine shops in Bozeman, Montana, USA (herein referred to as Shop 1 and Shop 2). MWF (S-500-CF, Hangsterfer's), a water-soluble fluid, was used at both shops. 
At the time of sample collection, this MWF had been in continuous use under routine fluid management practices for one year. Additionally, samples were collected from Shop 2 after a commonly followed industrial dumping, cleaning, and recharging process. Samples from this cleaned and recharged system were collected after one day of operation with new fluid. Samples for microbial community analysis from the MWF reservoir of CNC machines $(\sim 100 \mathrm{~mL})$ were filtered onto $47 \mathrm{~mm}$ Supor ${ }^{\circledR} 200$ PES membrane filters with a pore size of $0.2 \mu \mathrm{m}$ using a peristaltic pump. Biofilm from screens and shavings were scraped with a sterile spatula directly from CNC machine compartments into sterile $50 \mathrm{~mL}$ Falcon tubes. Mist from inside the CNC machine working chamber during operation in Shop 2 was collected onto $47 \mathrm{~mm}$ Supor® 200 PES membrane filters with a pore size of $0.2 \mu \mathrm{m}$. Membrane filters were placed into sterile tubes. All samples were stored at $-80^{\circ} \mathrm{C}$ until further processing.

\section{Extraction of nucleic acids, DNA amplification, and sequence analyses}

DNA was extracted using the PowerWater ${ }^{\circledR}$ DNA Isolation Kit (14900-S, MO BIO Laboratories) following the manufacturer's instructions. Extracted DNA was quantified using the high sensitivity Qubit DNA Assay Kit with a Qubit® Fluorometer (Thermo Fisher Scientific). The polymerase chain reaction (PCR; see Online Resource 1) was performed using primer pairs specific to the V3-V4 regions of the 16S rRNA gene. Primer complexes included the Illumina adaptor sequences followed by either the universal primers $341 \mathrm{~F}$ (5'acactctttccetacacgacgetcttccgatctCCTACGGGNGGCWGCAG-3') or 805R (5'gtgactggagttcagacgtgtgctcttccgatctGACTACHVGGGTATCTAATCC-3') (UW Biotechnology Center, Madison, WI, USA). PCR amplicons were submitted to the University of WisconsinMadison Biotechnology Center. A blank sample for the extraction procedure was included in the 
sequence libraries. Paired end, 250 bp sequencing was performed using an Illumina MiSeq Sequencer and a MiSeq 500 bp (v2) sequencing cartridge.

For joining the forward and reverse sequence libraries QIIME toolkit was used [7]. Quality refinement of contigs was performed using the Mothur platform v.1.34.4 [43; see Online Resource 1]. Operational taxonomic units (OTU) were defined at $\geq 97 \%$. Raw sequences libraries were deposited to the GenBank NCBI database under accession number SRA PRJNA398788.

Agents

Four natural agents, one enzyme, and two chemicals were tested for biofilm reduction and their effect on QSI. Emodin (E7881), gingerol (G1046), patulin (P1639), acylase I from porcine kidney (A3010), and the chemical agents furanone C-30 (53796) and phenyl disulfide (169021) were purchased from Sigma-Aldrich (Table 1). Garlic was extracted and purified adopting the protocol from Rasmussen et al. [39]. To evaluate the efficacy of QS inhibiting agents on biofilm reduction, a comparison was made with $\alpha$-amylase Bacillus amyloliquefaciens (Sigma-Aldrich A7595), an enzyme capable of degrading the extracellular polymeric substance (EPS) matrix of biofilms.

\section{Culture conditions}

Pseudomonas aeruginosa PAO1 (ATCC strain: 15692), a model biofilm forming organism, was grown in tryptic soy broth (TSB) at $37^{\circ} \mathrm{C}$ while shaking at $125 \mathrm{rpm}$ for 16 hours. Cultures were harvested and centrifuged at 10,000xg for $2 \mathrm{~min}$. Cell pellets were resuspended as applicable in either TSB or autoclaved municipal drinking water. Municipal drinking water was 
used for MWF assays, as the local machine shops use this water to dilute MWFs to the recommended operational concentration.

\section{Screening for compound applicability}

P. aeruginosa PAO1 enrichments were transferred into 96-well microtiter plates at an optical density (OD) of 0.03 at $600 \mathrm{~nm}$. Wells were supplemented with TSB (1X final concentration) and the agents shown in Table 1. Eight replicates per agent and control were tested. Quantitative analysis in microtiter plates was performed following the crystal violet method [35]. Plates were incubated at $37^{\circ} \mathrm{C}$ for 48 hours. At selected time points (i.e. 0, 24, 48 hours), plates were washed five times in deionized water (DIW) to remove planktonic cells. Wells were stained with $300 \mu \mathrm{L}$ of $1: 3$ diluted crystal violet solution for $20 \mathrm{~min}$ at $22^{\circ} \mathrm{C}$. Excess stain was washed off five times in DIW. Following washing, $300 \mu \mathrm{L}$ of $95 \%$ ethanol was added to each well, and de-colorization of the wells was allowed for $15 \mathrm{~min}$. Light absorbance data were read at $540 \mathrm{~nm}$ in a BioTek FL600 micro-plate reader.

\section{CDC Biofilm Reactors}

Compounds that demonstrated the highest percentage of biofilm reduction (i.e. $\alpha-$ amylase, patulin, and furanone C-30) were further investigated. CDC Biofilm Reactors (Biosurface Technologies, Bozeman, MT) were assembled with stainless-steel coupons and autoclaved for $30 \mathrm{~min}$. Each reactor contained $300 \mathrm{~mL}$ of $1 \mathrm{X}$ TSB medium, $2 \mathrm{~mL}$ of an overnight $P$. aeruginosa PAO1 culture, and a biofilm treatment agent (Table 1). Experiments were performed as batch reactors on a stir plate at $125 \mathrm{rpm}$ at $37^{\circ} \mathrm{C}$ for 48 hours. After 24 and 48 hours of incubation, one rod holding three stainless-steel coupons was removed from untreated 
and treated reactors. Coupons were dipped in $1 \mathrm{X}$ phosphate-buffered saline (PBS) to remove unattached, planktonic cells. Each coupon was then placed into sterile falcon tubes filled with 5 $\mathrm{mL}$ of $1 \mathrm{X}$ PBS and vortexed for $10 \mathrm{sec}$. Ten-fold dilution series were prepared in sterile 1X PBS. Five drops $(10 \mu \mathrm{L}$ each) per dilution were pipetted onto TSA plates [15], using an electronic pipette (ThermoLabsystems) for accuracy. Colony forming units (CFUs) of biofilm were enumerated after 16 hours of incubation at $37^{\circ} \mathrm{C}$. Resulting CFUs were $\log _{10}$-transformed. A log reduction (LR) was calculated for each experiment by subtracting the mean of the three logdensities for the treated coupons from the mean of the three log densities for the concurrent controls.

Multi-well plate assays with MWF

Overnight enrichments of $P$. aeruginosa PAO1 were harvested by centrifugation at $10,000 \mathrm{xg}$ for $2 \mathrm{~min}$, washed twice in autoclaved municipal drinking water, and transferred into 12-well microtiter plates (08-772-3A, Falcon ${ }^{\mathrm{TM}}$ Polystyrene Microplates) at an OD of 0.03 at 600 nm. Wells were supplemented with 7\% (final concentration) MWF in autoclaved municipal drinking water (i.e. recommended operational concentration, Hangsterfer's Laboratories Inc.) and the agents shown in Table 1. A stainless-steel coupon in each well served as the biofilm growth surface. Coupons were autoclaved for $30 \mathrm{~min}$ prior to the experiment. Agents and abiotic controls were tested in triplicate. Plates were incubated at $37^{\circ} \mathrm{C}$ for 48 hours. Biofilm formation was investigated after 24 and 48 hours by applying the drop-plating method described above.

\section{Microscopy}


Epi-fluorescence microscopy (Nikon Eclipse E-800) was performed to visualize biofilm on untreated and treated sample coupons. Coupons were pulled from CDC Biofilm Reactors after 48 hours and dipped in filter sterilized 1X PBS to remove planktonic cells from the coupon surface. Biofilm was stained with the fluorescent dye SYTO ${ }^{9}$ (L7012, Invitrogen by Thermo Fisher Scientific) in the dark at $22^{\circ} \mathrm{C}$ for $30 \mathrm{~min}$. Sample coupons were carefully rinsed with filter sterilized DIW prior to imaging. Samples were imaged under 60x magnification with the MetaVue version 7.8.13.0 (Molecular Devices, LLC.) software package. Randomly selected images $(\mathrm{n}=10)$ were taken per coupon. Interference between the MWF and epi-fluorescent signals prevented imaging of biofilm grown in MWF.

\section{Metabolite extraction}

Biofilm was grown on stainless-steel coupons in 12 -well plates in TSB or MWF at $37^{\circ} \mathrm{C}$ for 24 hours. Trials were prepared in triplicate for untreated controls, and patulin $(40 \mu \mathrm{M})$ and furanone C-30 $(75 \mu \mathrm{M})$ amendments. Triplicate stainless-steel coupons were pooled in sterile falcon tubes containing $6 \mathrm{~mL}$ of $1 \mathrm{X}$ PBS buffer and vortexed for $10 \mathrm{sec}$. Subsequently, cell pellets were collected by centrifugation at 10,000xg for $2 \mathrm{~min}$. Supernatant was discarded and cell pellets were re-suspended in $1 \mathrm{~mL} 1 \mathrm{X}$ PBS.

Metabolites were extracted according to a modified procedure from Carlson et al. [8; see Online Resource 1]. Samples were kept on ice at all time. Extracts were dried in a speed vacuum (Vacufuge plus, Eppendorf) for 2.5 hours, resuspended in $50 \%$ methanol and stored at $-80^{\circ} \mathrm{C}$ until liquid chromatography-mass spectrometry (LC-MS) was performed.

Metabolomic profiling and data processing 
Extracted metabolites were analyzed using an Agilent 1290 UPLC system connected to an Agilent 6538 Q-TOF mass spectrometer (Agilent, Santa Clara, CA). The samples were run in normal phase, using a Cogent Diamond Hydride HILIC $150 \times 2.1 \mathrm{~mm}$ column (MicroSolv, Eatontown, NJ). Output files were converted to MZxml format using the Masshunter Qualitative software provided with Agilent instruments (Agilent, Santa Clara, CA). Analysis of LC-MS data was done using the features and identification function in MZmine version 2.31 [36]. See Online Resource 1 for more details.

\section{Statistical analysis}

Linear mixed-effects models were applied to determine the repeatability of the untreated control log densities across experiments. Welch 2-sample $t$-tests were performed on log densities in single experiments to assess the efficiency of individual treatments compared to untreated controls. All pairwise comparisons of the mean LRs among the different agents, for each combination of media (MWF or TSB) and time points (24 or 48 hours), were performed by Welch $t$-tests with a Benjamini Hochberg correction. Linear mixed-effects models and follow-up $t$-tests using Kenward Roger degree of freedom approximation were applied to determine the repeatability of experiments [47]. Standard deviations (SD) for repeatability of $\leq 0.5$ are accepted [33]. The pairwise comparisons of LR were performed by Welch t-tests with a Bonferroni multiple comparison correction for each agent. All statistical analyses were performed using the statistical software R [37].

\section{Results}

Determination of microbial communities 
Quality refinement (including removal of blank OTUs and singletons) resulted in 98,162 $\pm 40,475$ sequences per library, which clustered into 46 OTUs. All samples collected tested positive for the presence of bacteria and were dominated by Pseudomonadales (60.2-99.7\%; Fig. 1). MWF was used at two different shops over a similar period; however, compared to Shop 1 dominated by Pseudomonadales (92 $\pm 4 \%$ ), the MWF circulation system in Shop 2 was colonized by both Pseudomonadales (74 $\pm 9 \%$ ) and Xanthomonadales (23 $\pm 9 \%)$. After dumping and cleaning the MWF circulation system at Shop 2, infection of the newly recharged MWF occurred within the first day, with Pseudomonadales driving recolonization.

\section{Efficacy of agents on biofilm reduction}

Various enzymes, natural, and chemical compounds were screened for their effect on biofilm formation (Table 1). Using crystal violet staining as an indicator for biofilm biomass, emodin $(10-1000 \mu \mathrm{M})$, garlic extract $(0.5 \%-15 \% \mathrm{v} / \mathrm{v})$, gingerol $(350 \mu \mathrm{M}), \alpha$-amylase $(\geq 10$ Units $\left.\mathrm{mL}^{-1}\right)$, acylase 1 from porcine kidney $\left(\sim 3.6-66.6\right.$ Units $\left.\mathrm{mL}^{-1}\right)$, and phenyl disulfide $(0.02-1 \mathrm{mM})$ showed no effect on eradication of $P$. aeruginosa PAO1 biofilms when compared to the untreated control. Conversely, patulin $(40 \mu \mathrm{M})$, furanone $\mathrm{C}-30(75 \mu \mathrm{M})$, and $\alpha$-amylase from $B$. amyloliquefaciens ( $\sim 3.4$ Units $\mathrm{mL}^{-1}$; herein referred to as $\alpha$-amylase) were effective in reducing biofilm formation (with reductions $\geq 50-80 \%$ ). These three agents were selected for further investigation of their effectiveness in reducing P. aeruginosa PAO1 biofilm formation.

When testing $P$. aeruginosa PAO1 biofilm grown in TSB in CDC batch reactors (Fig. 2a), patulin ( $p=0.034)$ and furanone $\mathrm{C}-30(p=0.002)$ demonstrated a statistically significant treatment effect after 24 hours when compared to an untreated reactor. Although incubation over 48 hours in TSB resulted in an increase in CFUs by 30.8 and $28.5 \%$ on average for patulin and 
furanone $\mathrm{C}-30$, respectively, differences were not statistically significant from the previous time point $(p \geq 0.160)$. Biofilm reduction efficiency between $\alpha$-amylase, a biofilm matrix degrading enzyme, and the two QS inhibitors were similar, and no significant differences were found after both 24 and 48 hours between compounds ( $p \geq 0.146$ ). Calculated standard deviations of untreated control $\log$ densities $(\mathrm{N}=18, \mathrm{SD}=0.245)$ suggests acceptable repeatability of the three treatments $[33,47]$. Treatment effects on $P$. aeruginosa PAO1 biofilm formation were also visualized by epi-fluorescent microscopy and SYTO ${ }^{\circledR} 9$ staining. Biofilm on stainless steel coupons treated with patulin and furanone C-30 was reduced in comparison to a denser growth on untreated coupons (Fig. 3). Green fluorescent cells indicated that the biofilm associated cells on the coupon surfaces were viable.

The same agents were applied to multi-well plate assays, but in MWF over a period of 48 hours (Fig. 2b). In 7\% MWF, both patulin and furanone C-30 led to significant biofilm reduction over 48 hours ( $p=0.001$ and $p=0.012$ ), respectively, decreasing CFUs by $63 \%$ and $76 \%$. In comparison, $\alpha$-amylase had little effect on biofilm reduction in MWFs after 48 hours when compared to the untreated control $(p=0.386)$ and differed significantly from the assays with QS inhibitors ( $\mathrm{p} \leq 0.043$ using Welch t-test). The untreated control log densities exhibited excellent repeatability across the three experiments Statistical analysis revealed the repeatability of these trials $(\mathrm{N}=18, \mathrm{SD}=0.159)$.

\section{Liquid chromatography-mass spectroscopy: Screening for quorum sensing signals}

LC-MS data were analyzed to identify the three major QS signaling molecules produced by $P$. aeruginosa PAO1. Putative identification showed the presence of C4-HSL (N-Butyryl-DLhomoserine lactone; expected $[\mathrm{M}+\mathrm{H}]^{+}: 172.10$, observed $\left.[\mathrm{M}+\mathrm{H}]^{+}: 172.09\right), 3-$ oxo-C12-HSL (N- 
(3-Oxo-dodecanoyl) homoserine lactone; expected $[\mathrm{M}+\mathrm{H}]^{+}: 298.20$, observed $[\mathrm{M}+\mathrm{H}]^{+}:$298.21), and PQS (Pseudomonas quinolone signal; expected $[\mathrm{M}+\mathrm{H}]^{+}: 259.16$, observed $[\mathrm{M}+\mathrm{H}]^{+}: 259.15$ ). Higher production of 3-oxo-C12-HSL was identified in P. aeruginosa PAO1 biofilm assays treated with furanone C-30 in MWF when compared to the untreated control (Fig. 4). Detection of C4-HSL and PQS by P. aeruginosa PAO1 growing in both TSB and MWF was reduced in the presence of QS inhibitors.

\section{Discussion}

Planktonic and biofilm samples collected from two local machine shops tested positive for microbial contamination and were dominated by Pseudomonadales (Fig. 1). Previous studies on microbial contaminants in MWFs confirm the dominance of this Order [3, 13]. Pseudomonas spp. may account for up to $\sim 90 \%$ of bacterial sequence libraries [10] with biomass as high as $>10^{8} \mathrm{CFUs} \mathrm{mL}^{-1}$ in some MWFs [27]. Of significance in the present study was the rapid recolonization after a commonly practiced dumping, cleaning, and recharge process at Shop 2. With residual microbes residing in inaccessible parts of MWF circulation systems escaping these hygiene practices, recolonization is an important issue in the MWF industry $[18,26,50]$. Most sequences classified within the Order Pseudomonadales were phylogenetically related to a single OTU. This OTU had $99 \%$ sequence identity to the $16 \mathrm{~S}$ rRNA gene of Pseudomonas stutzeri, a species frequently associated with biodegradation activity in MWFs [10, 13]. In preliminary experiments, $P$. stutzeri was unable to form stable biofilm in a CDC reactor. Thus, $P$. aeruginosa, another known MWF contaminant [10] was selected for QSI experiments. Specifically, P. aeruginosa POA1, a model strain for biofilm formation, was chosen [20]. 
Although biofilms in MWFs are pervasive, data concerning measures on biofilm removal are limited. Biocides are commonly applied to reduce the microbial load in MWFs; yet, a recent study investigating more than 60 machines operated with MWFs found no evidence for changes in the size of microbial populations in biofilms between untreated MWFs and those containing biocides [49]. Conversely, the QSI compounds patulin and furanone C-30 investigated in this study had a strong negative effect $(\mathrm{p} \leq 0.012)$ on biofilm formation of $P$. aeruginosa PAO1 on stainless-steel coupons in MWF, reducing CFUs in biofilm by $\sim 63 \%$ and $76 \%$ on average, respectively (Fig. 2). These efficiencies were higher when compared to previous reports on the inhibition of biofilm formation by patulin and furanone [21,53] and bracketed by efficacies reported for $\alpha$-amylase [17]. With biofilm formation being highly sensitive to the chemical and biological makeup of an environment, these comparisons should, nonetheless, be viewed conservatively. While $\alpha$-amylase, an enzyme that has been shown to significantly reduce biofilm formation and increase biofilm detachment [17], delayed initial biofilm development, it had no effect on biofilm reduction in MWF after 48 hours in the present study. It is noteworthy that as a mycotoxin, patulin could exhibit antibiotic properties and impair growth when administered at high concentrations. However, in both our work and others, inhibitory effects of patulin on cell growth of $P$. aeruginosa PAO1 were not detected for the applied concentrations [data not shown; $40]$.

It is well established that $P$. aeruginosa uses QS to collectively coordinate motility, virulence factors, and biofilm formation [28]. Its QS system consists of a multilayered, interconnected network, the LasI/R and RhlI/R system, with the binding of the autoinducers 3oxo-C12-HSL and C4-HSL to their cognate LasR and RhlR regulatory proteins, respectively, activating the transcription of QS controlled genes [28]. LasR not only regulates the expression 
of C4-HSL, but also genes involved in the synthesis of a large array of 4-hydroxy-2alkylquinoline (HAQ) QS molecules. The best characterized HAQ is PQS, which is directly involved in cell-to-cell signaling [28].

While patulin and furanone C-30 do not interfere with the synthesis of 3-oxo-C12-HSL, per se, in silico analysis shows a high affinity of patulin and furanone C-30 to the LasR receptor site [45], the protein that regulates genes involved in the synthesis of C4-HSL and PQS. Patulin mimics the lactone moiety of HLSs with the ability to bind to LasR via H-bonds. Furanone C-30, on the other hand, blocks the formation of the hydrophobic core of LasR and the breaking of internal H-bonds further destabilizes the protein [4]. The expression of C4-HSL and PQS in this study was consistently lower in all samples treated with patulin and furanone C-30, particularly in MWFs. Most importantly, these two QS molecules are critical to biofilm development as they activate, among others, genes encoding elastase, rhamnolipids, phenazines, carbohydrate-binding lectins, and EPS secretion [29, 34, 38, 52]; genes which are downregulated by patulin and furanone C-30 $[14,39]$. While QS signaling molecules are produced and released at a low baseline level, the activation of the QS system is auto-regulated and depends on a threshold concentration of signaling molecules related to cell density [28]. In fact, an excess of exogenous signaling molecules is insufficient to induce a QS response when cell densities are low [14]. In agreement with these reports, the detection of putatively identified QS molecules (i.e. 3-oxoC12-HSL, C4-HSL, and PQS) corresponded with fewer CFUs in the present study (Fig. 3) and was, with one exception, lower in samples treated with QS inhibitors (Fig. 4).

By manipulating the QS circuitry on a transcriptional level and by interfering with the synthesis and accumulation of QS molecules, patulin and furanone C-30 may have affected a cascade of genes, including those involved in biofilm formation. The application of these QS 
inhibitors has also been shown to render $P$. aeruginosa biofilm more susceptible to biocide treatment $[14,39]$. With biocides commonly used to combat microbial growth in MWFs, their combination with QS inhibitor agents awaits investigation for preventing biofilm formation. Further, while patulin and furanone C-30 were administrated only at the beginning of the 48 hours of incubation, improved biofilm reduction may be achieved by periodic amendments of QS inhibitors [14, 51]. A recent review of environmental applications of QSI predicts that in the future wastewater treatment will indispensably rely on QS-based manipulations (25); findings that may greatly encourage research on the applicability of QSI in other systems. As such, data presented herein highlight QSI as a promising approach for battling microbial contaminants in MWFs and warrants future fundamental and large-scale trials on QS-QSI to optimize alternative strategies that could replace or supplement the usage of biocides in MWFs. Brinksmeier et al. (5) provided an overview of the development of MWF, the underlying mechanisms behind various additives, and the state of the field regarding improvements to MWF performance. Aspects including complex chemistry, regulatory requirements, and effect of such additives on MWF formulations would need to be addressed before QSI could be fully implemented into the day to day operations of the real-world environment.

The authors declare no competing financial interest.

\section{Online Resource 1}

Methodological details for polymerase chain reaction, quality screening of sequences, metabolite extraction, and metabolomic profiling and data processing. 


\section{Figure Legend:}

Fig. 1 Relative abundance of the microbial contamination (Order level) present in CNC equipment at two machine shops (Bozeman, MT)

Fig. 2 Effect of quorum sensing inhibitors on P. aeruginosa PAOl biofilm formation in (a) $1 \mathrm{X}$ TSB in CDC batch reactors, and (b) 7\% (final concentration) water soluble MWF in 12-well plates. Data points represent CFU biofilm reduction (\%) at 24 and 48 hours. Error bars indicate $95 \%$ confident intervals. Percent reductions that met significant levels are shown as $* \leq 0.05, * *$ $\leq 0.01, * * * \leq 0.001$

Fig. 3 Epi-fluorescent images of $P$. aeruginosa PAO1 biofilm stained with SYTO® 9 on stainless-steel coupons after 48 hours in TSB. Biofilm was imaged under 60x magnification.

Scale bar $=20 \mu \mathrm{m}$. (a) CDC control reactor, (b) patulin and (c) furanone C-30 treatment

Fig. 4 Relative production of putatively identified QS molecules found in P. aeruginosa PAO1 biofilm in the presence of QSI after 24 hours in TSB and MWF. The baseline represents detection of putatively identified QS molecules identified in the untreated controls 


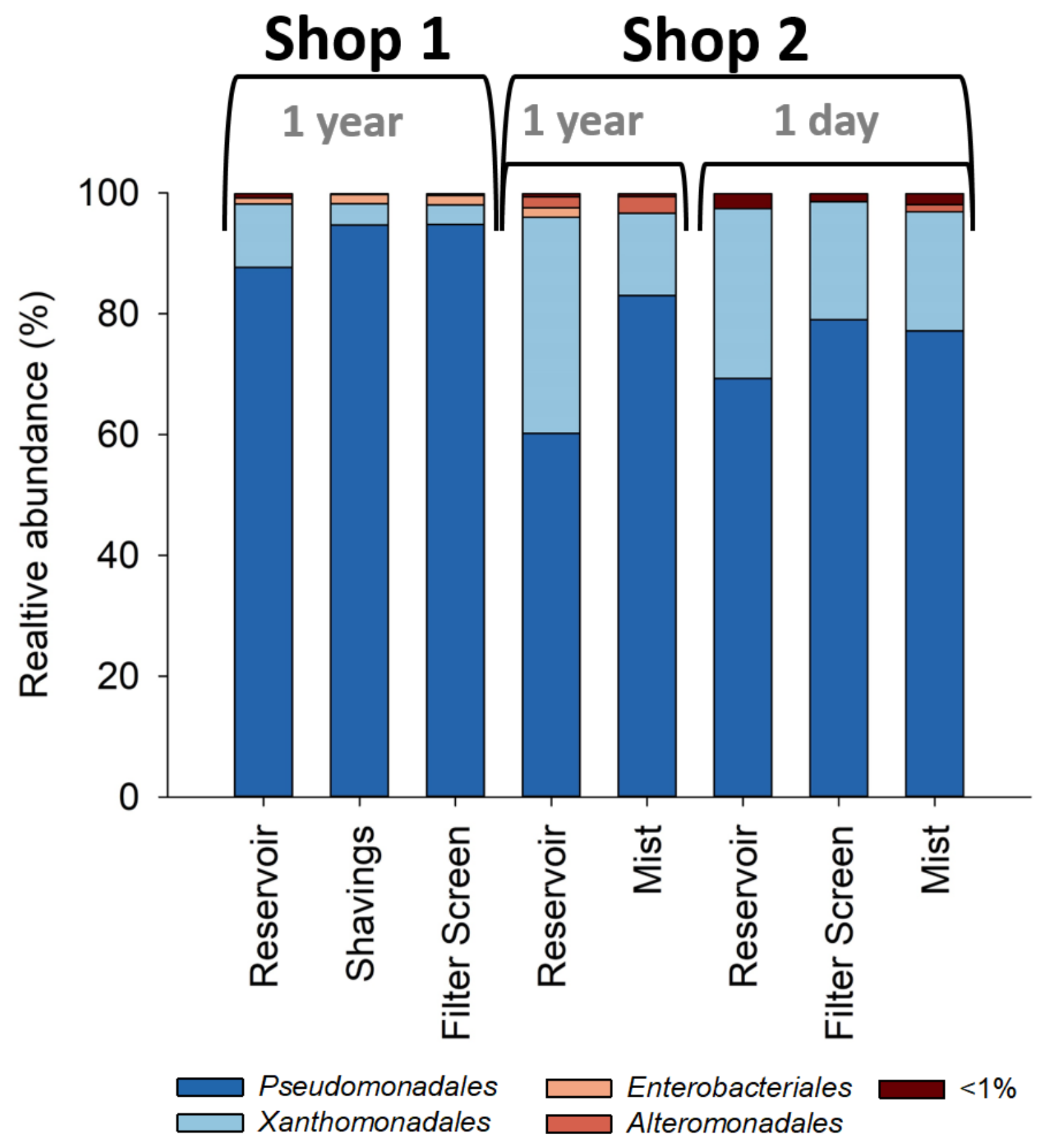

Fig. 1 


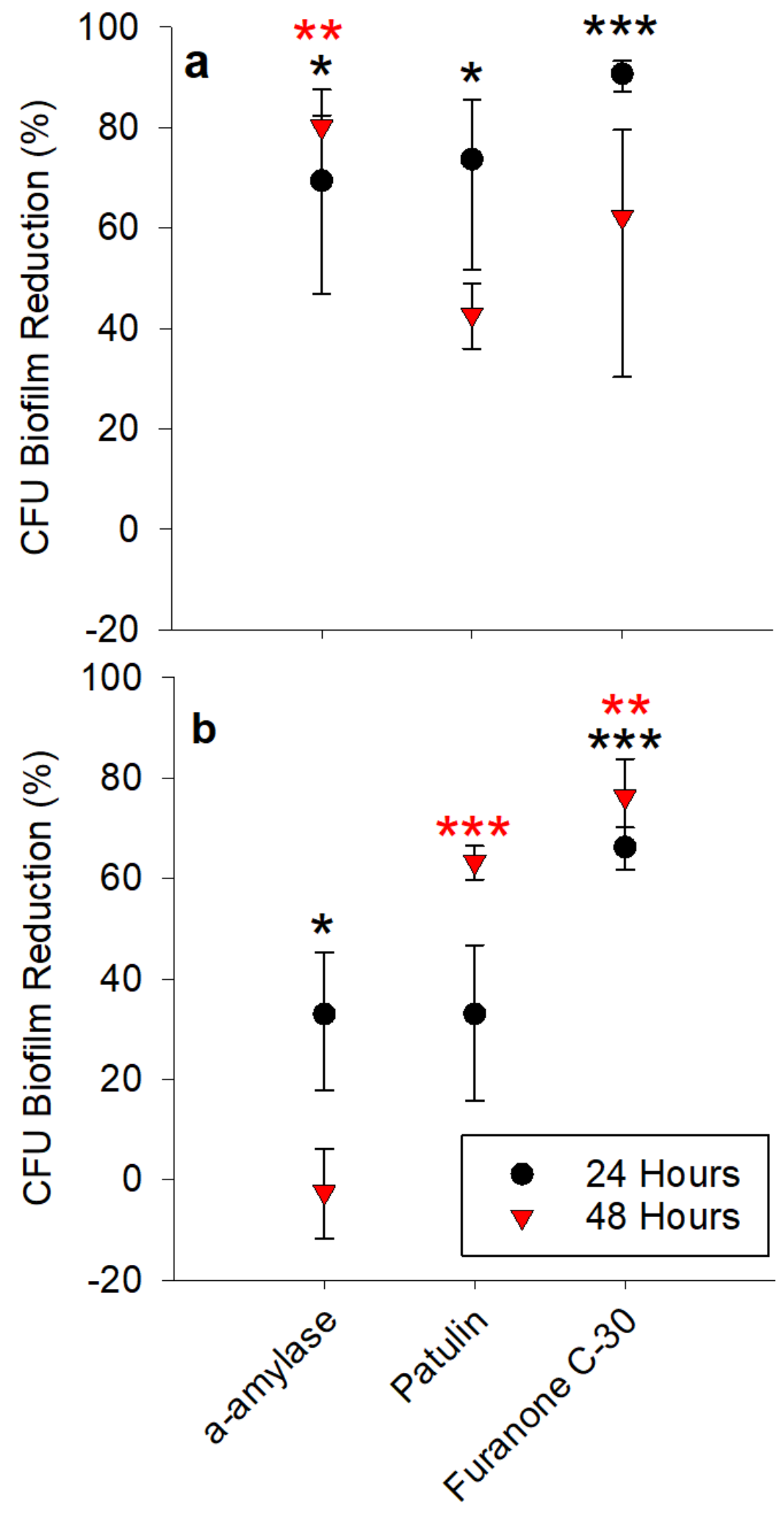

Fig. 2 


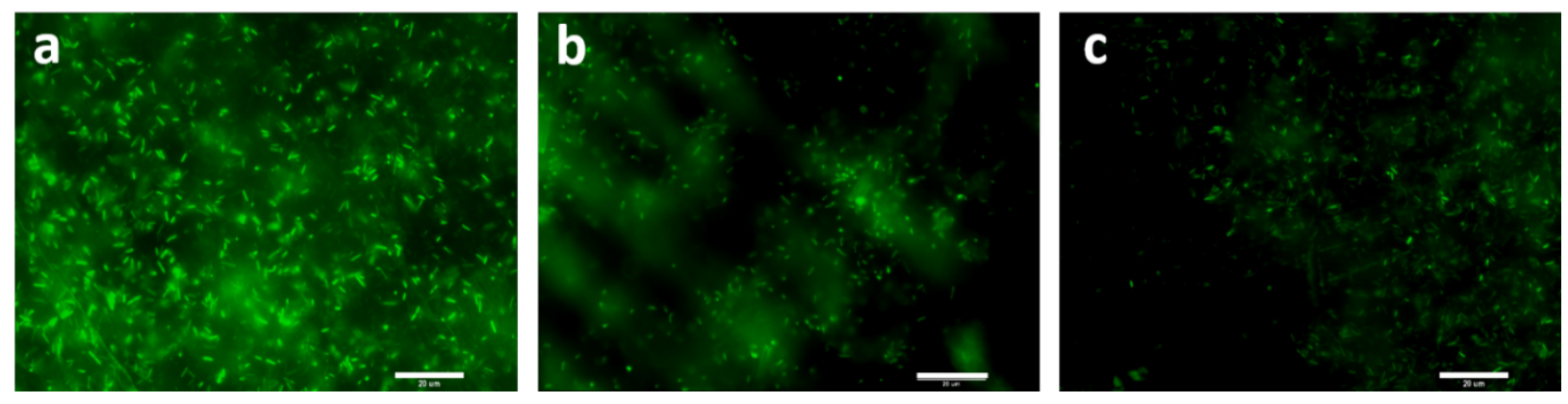

Fig. 3 


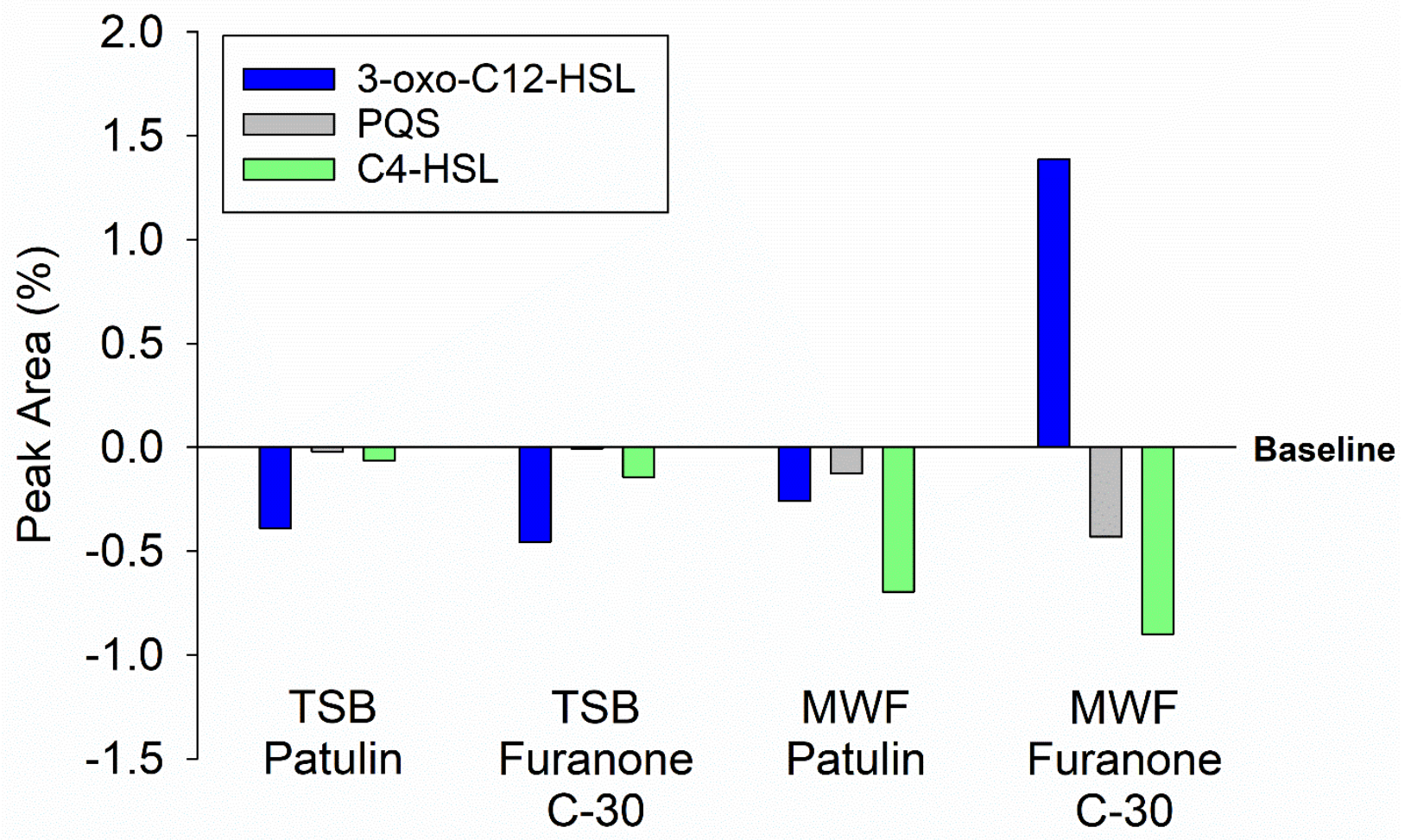

Fig. 4 
Table 1: Compounds tested for reducing $P$. aeruginosa PAO1 biofilm formation grown in 96 well microtiter plates in TSB. Efficacy is presented as percent biofilm reduction in comparison to the spectrophotometric measurements on crystal violet strained biomass in untreated controls. Asterisk identifies agents selected for QSI assays.

\begin{tabular}{|c|c|c|c|c|}
\hline \multirow[b]{2}{*}{ Types } & \multirow[b]{2}{*}{ Agent } & \multicolumn{3}{|c|}{ \% Biofilm Reduction } \\
\hline & & $\geq 80$ & $\geq \mathbf{5 0}$ & No Effect \\
\hline \multirow[t]{4}{*}{ Natural } & Emodin $(10-1000 \mu \mathrm{M})$ & & & $\mathrm{x}$ \\
\hline & Garlic extract $(0.5-15 \% \mathrm{v} / \mathrm{v})$ & & & $\mathrm{x}$ \\
\hline & Gingerol (350 uM) & & & $\mathrm{x}$ \\
\hline & Patulin (40 uM)* & $\mathrm{x}$ & & \\
\hline \multirow[t]{3}{*}{ Enzymes } & $\alpha$-amylase $B$. amyloliquefaciens $\left(3.4 \mathrm{U} \mathrm{mL}^{-1}\right)^{*}$ & $\mathrm{x}$ & & \\
\hline & $\alpha$-amylase $B$. amyloliquefaciens $\left(\geq 10 \mathrm{U} \mathrm{mL}^{-1}\right)$ & & & $\mathrm{x}$ \\
\hline & Acylase 1 from porcine kidney $\left(\sim 3.6-66.6 \mathrm{U} \mathrm{mL}^{-1}\right)$ & & & $\mathrm{x}$ \\
\hline Synthetic & Furanone C-30 (75 uM)* & & $\mathrm{x}$ & \\
\hline Chemicals & Phenyl disulfide (0.02-1 mM) & & & $\mathrm{x}$ \\
\hline
\end{tabular}




\section{References Cited}

1. Ashraf MA, Ullah S, Ahmad I, Qureshi AK, Balkhair KS, Abdur Rehman M (2014) Green biocides, a promising technology: current and future applications to industry and industrial processes. J Sci Food Agric 94:388-403.

2. Azimi Y, Thompson IP (2017) Phosphorus depletion as a green alternative to biocides for controlling biodegradation of metalworking fluids. Environ Sci Technol 51:5695-5702.

3. Baecker AA, Leroux K, Vonholy A (1989) Microbiological contaminants of metalworking fluids in service. S Afr J Sci 1989 85:293-295.

4. Bottomley MJ, Muraglia E, Bazzo R, Carfì A (2007) Molecular insights into quorum sensing in the human pathogen $P$. aeruginosa from the structure of the virulence regulator LasR bound to its autoinducer. J Biol Chem 282:13592-13600.

5. Brinksmeier E, Meyer D, Huesmann-Cordes AG, Herrmann C (2015). Metalworking fluids - mechanisms and performance. CIRP Annals 64:605-628.

6. Burge PS (2016) Hypersensitivity pneumonitis due to metalworking fluid aerosols. Curr Allergy Asthma Rep 16:59.

7. Caporaso JG, Kuczynski J, Stombaugh J, Bittinger K, Bushman FD, Costello EK, Fierer N, Pena AG, Goodrich JK, Gordon JI, Huttley GA, Kelley ST, Knights D, Koenig JE, Ley RE, Lozupone CA, McDonald D, Muegge BD, Pirrung M, Reeder J, Sevinsky JR, Turnbaugh PJ, Walters WA, Widmann J, Yatsunenko T, Zaneveld J, Knight R (2010) QIIME allows analysis of high-throughput community sequencing data. Nat Methods $7: 335$. 
8. Carlson AK, Rawle RA, Adams E, Greenwood MC, Bothner B, June RK (2018)

Application of global metabolomic profiling of synovial fluid for osteoarthritis biomarkers. Biochem Biophys Res Commun 499:82-88.

9. Ding X, Yin B, Qian L, Zeng Z, Yang Z, Li H, Lu Y, Zhou S (2011). Screening for novel quorum-sensing inhibitors to interfere with the formation of Pseudomonas aeruginosa biofilm. J Med Microbiol 60:1827-1834.

10. Di Maiuta N, Rüfenacht A, Küenzi P (2017) Assessment of bacteria and archaea in metalworking fluids using massive parallel 16S rRNA gene tag sequencing. Lett Appl Microbiol 65:266-273.

11. Falkinham JO (2009) Effects of biocides and other metal removal fluid constituents on Mycobacterium immunogenum. Appl Environ Microbiol 75:2057-2061.

12. Fong J, Zhang C, Yang R, Boo ZZ, Tan SK, Nielsen TE, Givskov M, Liu XW, Bin W, Su H, Yang L (2018). Combination therapy strategy of quorum quenching enzyme and quorum sensing inhibitor in suppressing multiple quorum sensing pathways of $P$. aeruginosa. Sci Rep 8:1155.

13. Gilbert Y, Veillette M, Duchaine C (2010) Metalworking fluids biodiversity characterization. J Appl Microbiol 108:437-449.

14. Hentzer M, Wu H, Andersen JB, Riedel K, Rasmussen TB, Bagge N, Kumar N, Schembri MA, Song Z, Kristoffersen P, Manefield M (2003) Attenuation of Pseudomonas aeruginosa virulence by quorum sensing inhibitors. EMBO J 22:38033815.

15. Herigstad B, Hamilton M, Heersink J (2001) How to optimize the drop plate method for enumerating bacteria. J Microbiol Methods 44:121-129. 
16. Kalia VC, Patel SK, Kang YC, Lee JK (2019). Quorum sensing inhibitors as antipathogens: biotechnological applications. Biotechnol Ad 37:68-90.

17. Kalpana BJ, Aarthy S, Pandian SK (2012) Antibiofilm activity of $\alpha$-amylase from Bacillus subtilis S8-18 against biofilm forming human bacterial pathogens. Appl Biochem Biotechnol 167:1778-1794.

18. Kapoor R, Selvaraju SB, Yadav JS (2014) Extended tracking of the microbial community structure and dynamics in an industrial synthetic metalworking fluid system. FEMS Microbiol Ecol 87:664-677.

19. Katebian L, Gomez E, Skillman L, Li D, Ho G, Jiang SC (2016) Inhibiting quorum sensing pathways to mitigate seawater desalination RO membrane biofouling. Desalination 393:135-143.

20. Kato J, Kim HE, Takiguchi N, Kuroda A, Ohtake H (2008) Pseudomonas aeruginosa as a model microorganism for investigation of chemotactic behaviors in ecosystem. J Biosci Bioeng 106:1-7.

21. Kim HS, Lee SH, Byun Y, Park HD (2015) 6-Gingerol reduces Pseudomonas aeruginosa biofilm formation and virulence via quorum sensing inhibition. Sci Rep 5:8656.

22. Lee S, Park SK, Kwon H, Lee SH, Lee K, Nahm CH, Jo SJ, Oh HS, Park PK, Choo KH, Lee CH (2016) Crossing the border between laboratory and field: bacterial quorum quenching for anti-biofouling strategy in an MBR. Environ Sci Technol 50:1788-1795.

23. Lodders N, Kämpfer P (2012) A combined cultivation and cultivation-independent approach shows high bacterial diversity in water-miscible metalworking fluids. Syst Appl Microbiol 35:246-252. 
24. Lucchesi EG, Eguchi SY, Moraes ÂM (2012) Influence of a triazine derivative-based biocide on microbial biofilms of cutting fluids in contact with different substrates. J Ind Microbiol Biotechnol 39:743-748.

25. Maddela NR, Binbin S, Yuan S, Zhou Z, Villamar-Torres R, Meng F (2019). Roles of quorum sensing in biological wastewater treatment: A critical review. Chemosphere 221:616-629.

26. Marchand G, Lavoie J, Racine L, Lacombe N, CloutierY, Bélanger É, Lemelin C, Desroches J (2010 Evaluation of bacterial contamination and control methods in soluble metalworking fluids. J Occup Environ Hyg 7:358-366.

27. Mattsby-Baltzer I, Sandin M, Ahlström B, Allenmark S, Edebo M, Falsen E, Pedersen K, Rodin N, Thompson RA, Edebo L (1989) Microbial growth and accumulation in industrial metal-working fluids. Appl Environ Microbiol 55:2681-2689.

28. Miller MB, Bassler BL (2001) Quorum sensing in bacteria. Annu Rev Microbiol 55:165199.

29. Nadell CD, Xavier JB, Levin SA, Foster KR (2008) The evolution of quorum sensing in bacterial biofilms. PLoS Biol 6:e14.

30. Oh HS, Yeon KM, Yang CS, Kim SR, Lee CH, Park SY, Han JY, Lee JK (2012) Control of membrane biofouling in MBR for wastewater treatment by quorum quenching bacteria encapsulated in microporous membrane. Environ Sci Technol 46:4877-4884.

31. O'Loughlin CT, Miller LC, Siryaporn A, Drescher K, Semmelhack MF, Bassler BL (2013). A quorum-sensing inhibitor blocks Pseudomonas aeruginosa virulence and biofilm formation. PNAS 110:17981-17986. 
32. Papenfort K, Bassler BL (2016). Quorum sensing signal-response systems in Gramnegative bacteria. Nat Rev Microbiol 14:576.

33. Parker A, Hamilton M (2011) Assessing resemblance, repeatability and reproducibility for quantitative methods Center for Biofilm Engineering, KSA-SM-10. http://www biofilm montana edu/content/ksa-sm-10.

34. Passos da Silva D, Schofield MC, Parsek MR, Tseng BS (2017) An update on the sociomicrobiology of quorum sensing in gram-negative biofilm development. Pathogens $6: 51$.

35. Pitts B, Hamilton MA, Zelver N, Stewart PS (2003) A microtiter-plate screening method for biofilm disinfection and removal. J Microbiol Methods 54: 269-276.

36. Pluskal T, Castillo S, Villar-Briones A, Orešič M (2010) MZmine 2: modular framework for processing, visualizing, and analyzing mass spectrometry-based molecular profile data. BMC Bioinformatics 11:395.

37. R Core Team R: A language and environment for statistical computing R Foundation for Statistical Computing, Vienna, Austria 2017. https://wwwR-projectorg/.

38. Ramos I, Dietrich LE, Price-Whelan A, Newman DK (2010) Phenazines affect biofilm formation by Pseudomonas aeruginosa in similar ways at various scales. Res Microbiol 161:187-191.

39. Rasmussen TB, Bjarnsholt T, Skindersoe ME, Hentzer M, Kristoffersen P, Köte M, Nielsen J, Eberl L, Givskov M (2005) Screening for quorum-sensing inhibitors (QSI) by use of a novel genetic system, the QSI selector. J Bacteriol 187:1799-1814. 
40. Rasmussen TB, Skindersoe ME, Bjarnsholt T, Phipps RK, Christensen KB, Jensen PO, Andersen JB, Koch B, Larsen TO, Hentzer M, Eberl L (2005) Identity and effects of quorum-sensing inhibitors produced by Penicillium species. Microbiol 151:1325-1340.

41. Reuter K, Steinbach A, Helms V (2016). Interfering with bacterial quorum sensing. PMC-S13209.

42. Shaaban M, Elgaml A, Habib ES (2019). Biotechnological applications of quorum sensing inhibition as novel therapeutic strategies for multidrug resistant pathogens. Microbial Pathogenesis 127:138-143.

43. Schloss PD, Gevers D, Westcott SL (2011) Reducing the effects of PCR amplification and sequencing artifacts on 16S rRNA-based studies. PloS One 6:e27310.

44. Siddiqui MF, Rzechowicz M, Harvey W, Zularisam AW, Anthony GF (2015). Quorum sensing based membrane biofouling control for water treatment: A review. J Water Process Engineering 7:112-22.

45. Skindersoe ME, Alhede M, Phipps R, Yang L, Jensen PO, Rasmussen TB, Bjarnsholt T, Tolker-Nielsen T, Høiby N, Givskov M (2008) Effects of antibiotics on quorum sensing in Pseudomonas aeruginosa. Antimicrob Agents Chemother 52:3648-3663.

46. Ta C, Arnason J (2016). Mini review of phytochemicals and plant taxa with activity as microbial biofilm and quorum sensing inhibitors. Molecules 21:29.

47. Tilt N, Hamilton MA (1999) Repeatability and reproducibility of germicide tests: a literature review. J AOAC Int 82:384-389.

48. Trafny E (2013) Microorganisms in metalworking fluids: current issues in research and management. Int J Occup Med Environ Health 26:4-15. 
49. Trafny EA, Lewandowski R, Kozłowska K, Zawistowska-Marciniak I, Stępińska M (2015) Microbial contamination and biofilms on machines of metal industry using metalworking fluids with or without biocides. Int Biodeterior Biodegradation 99:31-38.

50. Veillette M, Thorne PS, Gordon T, Duchaine C (2004) Six month tracking of microbial growth in a metalworking fluid after system cleaning and recharging. Ann Occup Hyg 48:541-546.

51. Wu H, Song Z, Hentzer M, Andersen JB, Molin S, Givskov M, Høiby N (2004) Synthetic furanones inhibit quorum-sensing and enhance bacterial clearance in Pseudomonas aeruginosa lung infection in mice. J Antimicrob Chemother 53:1054-1061.

52. Yu H, He X, Xie W, Xiong J, Sheng H, Guo S, Huang C, Zhang D, Zhang K (2014) Elastase LasB of Pseudomonas aeruginosa promotes biofilm formation partly through rhamnolipid-mediated regulation. Can J Microbiol 60:227-235.

53. Zhou J, Bi S, Chen H, Chen T, Yang R, Li M, Fu Y, Jia AQ (2017) Anti-biofilm and antivirulence activities of metabolites from Plectosphaerella cucumerina against Pseudomonas aeruginosa.Front Microbiol 8:769. 